\title{
La alfabetización informacional en las universidades españolas. Niveles de incorporación a partir de la información publicada en los sitios web de sus bibliotecas-CRAI ${ }^{1}$
}

\author{
Alejandro Uribe-Tirado*, Astrid Girlesa Uribe**
}

Resumen: La formación en competencias informacionales o alfabetización informacional es uno de los retos actuales de las bibliotecas-CRAI (Centros de Recursos para el aprendizaje y la investigación) ante las posibilidades de acceso a enormes recursos electrónicos de información que facilitan los medios digitales, que obligan a conocer y aplicar mejores criterios de selección y evaluación para recuperar la de mayor calidad y pertinencia según las necesidades de información. Ante esta situación, las bibliotecas-CRAI universitarias iberoamericanas han ido poco a poco incorporando esta formación bien sea desde programas-cursos directos ofrecidos desde la biblioteca-CRAI o mediante el trabajo colaborativo con docentes y facultades en currícula de distintas universidades como un todo o en disciplinas específicas. Este trabajo utilizando el análisis de contenidos, hace una revisión de la información que presentan los sitios Web de las bibliotecas-CRAI de las instituciones de educación superior españolas (IES) sobre esta actividad de formación y genera una descripción y clasificación de los niveles de incorporación de las competencias informacionales que estarían presentando.

Palabras clave: Alfabetización informacional, análisis de contenidos, bibliotecas universitarias, competencias informacionales, España, sitios Web.

\section{Information literacy in Spanish universities. Degree of implementation based on library-LRC Website information}

Abstract: Training in information competencies or information literacy is one of the current challenges facing academic libraries-LRC (Learning Resources Center). The possibilities for accessing vast quantities of electronic information resources require a better understanding and application of selection and assessment criteria for retrieving high quality information of the greatest relevance to information needs. Given this situation, Ibero-American university libraries-LRCs (Latin-America, Spain and Portugal) have

${ }^{1}$ Este artículo se enmarca en la investigación: "Lecciones aprendidas en programas de Alfabetización Informacional en universidades de Iberoamérica» (Uribe-Tirado, A. 2010-...; Tutora: María Pinto Molina). Para el caso español, se contó con la participación de la bibliotecóloga Astrid Girlesa Uribe, como parte de su práctica académica de apoyo a una investigación, como requisito para obtener su título de grado.

* Universidad de Antioquia (Medellín-Colombia). Correo-e: auribe@bibliotecologia.udea.edu.co.

* Universidad de Granada (Granada-España) - AUIP. Correo-e: auribe@correo.ugr.es.

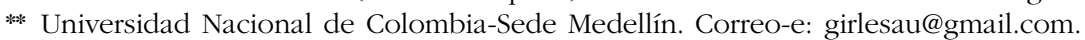

Recibido: 11-07-2011; 2. ${ }^{a}$ versión: 09-09-2011; aceptado: 21-02-2012. 
been slowly incorporating information literacy training, such as direct training programs offered by the library or working collaboratively with teaching staff and academic departments concerning curricula for the entire university as a whole or for specific disciplines. Using content analysis, the article reviews information presented on the websites of Spanish university libraries-LRCs about this core training activity, as well as describing and classifying the degree to which information literacy has been implemented.

Keywords: Content analysis, information competencies, information literacy, Spain, university libraries, Web sites.

\section{Introducción}

La formación en competencias informacionales o alfabetización informacional - ALFIN - es una temática que desde mediados de los años ochenta, con sus debidas precisiones y adaptaciones sobre su alcance y sus aspectos terminológicos, conceptuales y teóricos ha venido desarrollándose en Iberoamérica ${ }^{2}$ y específicamente en España ${ }^{3}$ desde el contexto de las bibliotecas, especialmente desde las de nivel universitario, que es donde en términos generales ha habido más avances y desde donde se marcan las futuras tendencias (Pinto-Molina y UribeTirado, 2011).

Tras estos más de 25 años de precisiones y adaptaciones que han llevado al paso de la instrucción bibliográfica y la formación de usuarios tradicional hasta llegar hoy al paradigma de la formación en ALFIN, se han dado diferentes procesos de avance, períodos de desarrollo (Uribe-Tirado, 2010a-b): «Pre-Inicio (19851994); Inicio (1995-1999); Pre-Avance (2000-2003); Avance (2004-2007) y PrePosicionamiento (2008-...), además de la visualización futura, según se vayan dando determinados procesos y resultados de éxito, de un $6 .^{\circ}$ período: Posicionamiento (2012 o 2013...)».

Considerando estos procesos-períodos generales, un aspecto clave es identificar de manera específica cómo se están llevando a cabo al interior de cada universidad, de cada Institución de Educación Superior (IES) la incorporación de esta formación en ALFIN, para con ello, poder identificar lecciones aprendidas y buenas prácticas que permitan a otras Universidades-IES de nuestro contexto Iberoamericano, con sus debidas adaptaciones, poder mejorar y avanzar en sus programas de formación en competencias informacionales.

Para dicha identificación, una fuente de información clave son los sitios Web de las bibliotecas-CRAI universitarias, ya que especialmente desde la última década, es sin duda este medio, el que más impacto está teniendo en la divulgación e interacción de sus servicios de información-documentación (Detlor y Lewis, 2006; Gardner y otros, 2008; entre otros), donde está incluida la formación.

\footnotetext{
${ }^{2}$ Ver recopilación de autores iberoamericanos en el repositorio-Wiki ALFIN/Iberoamérica: http://alfiniberoamerica.wikispaces.com/.

3 Ver recopilación de autores españoles en el repositorio-Wiki ALFIN/Iberoamérica: http://alfiniberoamerica.wikispaces.com/España.
} 
Específicamente con los servicios de formación (Ferreira-Gomes y otros, 2010), por la divulgación e interacción que permiten en dos vías:

- Para los públicos internos: estudiantes, profesores, investigadores y/o directivos de su institución a quienes van dirigidos sus cursos-tutoriales de formación en ALFIN.

- Para los públicos externos: bibliotecólogos, bibliotecarios, profesores y/o directivos de otras IES que quieren conocer la oferta formativa de esa institución y su biblioteca-CRAI para realizar procesos de benchmarking, de trabajo cooperativo interinstitucional, de consorcios formativos, etc.

Por tanto, este trabajo presenta a continuación un análisis del caso particular de España, tras el análisis de los sitios web de las bibliotecas-CRAI de sus Universidades-IES, y según la información que presentan, los niveles en que se encontrarían en la incorporación de ALFIN desde una perspectiva de UniversidadIES alfabetizada informacionalmente (Webber y Johnston, 2006).

\section{Niveles de incorporación de ALFIN en las universidades-IES}

Desde nuestra perspectiva, retomando trabajos previos (Uribe-Tirado, 20102011) que recopilan propuestas de diferentes autores, una Universidad-IES o dependencia al interior de la misma que deba liderar procesos-programas de formación en ALFIN (Sistema de Bibliotecas, Biblioteca, CRAI, etc.) puede categorizarse, haciendo una generalización de los distintos contextos, en 4 categorías según el grado de incorporación: Universidades-IES comprometidas/en crecimiento/iniciando/desconocedoras . $^{4}$.

Estos grados de incorporación de la formación en ALFIN pueden sintetizarse e identificarse cuando se analizan los sitios Web de las bibliotecas de las Universidades-IES teniendo en cuenta diferentes elementos (variables), que se resumen en:

Se detecta en el sitio web una sección o información concreta sobre la formación en ALFIN: (Sí - No).

Si la respuesta es No, conllevaría directamente a la categoría de Desconocedora; si la respuesta es Sí, conllevaría al análisis y clasificación en las categorías según la información que presentan (7 macrovariables a valorar):

1. Definición del programa: Misión, Visión, Objetivos, Plan operativo, Plan pedagógico.

\footnotetext{
${ }^{4}$ Ver esta clasificación según el nivel de incorporación de ALFIN en:

http://alfincolombia.blogspot.com/2010/02/la-alfabetizacion-informacional-en-la.html.
} 
2. Fundamentación del programa: Definición de ALFIN, Modelo pedagógicodidáctico de ALFIN y Estándar-Indicadores de ALFIN asumidos.

3. Estructuración del programa: Cursos ofrecidos, Modalidad de los cursos, Incorporación de las TIC, Web 2.0 y de ambientes virtuales de aprendizaje, Presencia curricular o extracurricular de los cursos.

4. Evaluación del programa: Indicadores de gestión, Indicadores de resultados de aprendizaje, Procesos de mejoramiento continuo.

Además de otra información complementaria, como:

5. Publicaciones sobre este tema y la experiencia de la biblioteca y del programa de ALFIN: artículos, ponencias, recursos Web 2.0.

6. Participación en grupos y redes de trabajo sobre esta temática: redes de bibliotecas universitarias, colectivos de bibliotecólogos/bibliotecarios, etc.

7. Participación en medios y recursos de divulgación y aprendizaje de esta temática: listas de discusión, blogs, wikis, twitters, boletines, comunidades virtuales, etc.

De estas 7 variables, es especialmente la referente a Estructuración del programa la que puede dar más cuenta del grado de incorporación que ALFIN tendría en una Universidad-IES y su Dependencia a cargo (Biblioteca-CRAI, etc.), ya que la presencia de determinados Cursos-Tutoriales permite visualizar con mayor facilidad el alcance que tiene el programa (facultades, titulaciones, número de estudiantes, etc.) y si se trabajan las 5, 6 o 7 competencias que presentan los modelos-estándares universitarios de ALFIN (según el que se haya elegido seguir).

Esto conlleva a una correlación de esta variable específica, que se puede visualizar desde los sitios Web, con las 4 categorías de incorporación que dan cuenta del proceso de desarrollo de esta temática, que han sido avaladas por expertos internacionales (Sonntag, 2011) y comparte la visión de documentos y propuestas como EMPATIC de la Unión Europea (2009) que cita al marco Australiano-Neozelandés -ANZIL_- considerando el paso al interior de las Universidad-IES de la instrucción bibliográfica y a la formación de usuarios tradicional hasta llegar hoy a la ALFIN en lo curricular:

\section{COMPROMETIDAS:}

- Alfabetización informacional. Nivel 2: cursos desde la biblioteca para formar en competencias informacionales: lo instrumental + aprendizaje para toda la vida + pensamiento crítico; y cursos/módulos específicos inmersos oficialmente en los currículos de distintos programas académicoscarreras para formar de manera transversal y disciplinar en esas competencias. 


\section{EN CRECIMIENTO:}

- Alfabetización informacional. Nivel 1: cursos desde la biblioteca para formar en competencias informacionales: lo instrumental + aprendizaje para toda la vida + pensamiento crítico.

\section{INICIANDO:}

- Formación de usuarios. Nivel 1: capacitación en servicios generales de la biblioteca y algunos cursos — muy instrumentales - para búsqueda de información: utilización de catálogos/bases de datos, aunque se comienza a analizar la necesidad de cambio de esta formación tradicional y a trabajar las demás competencias.

\section{DESCONOCEDORAS:}

- Formación de usuarios. Nivel 2: solo capacitación para el uso del catálogo.

- No bay presencia de ningún tipo de formación-capacitación.

Es importante indicar, al tener en cuenta este marco metodológico y teóricoconceptual para visualizar los posibles niveles de incorporación de ALFIN en las bibliotecas-CRAI universitarias, que esta categorización es una aproximación desde la información que conllevan los sitios web, pero en ningún momento implica que categorizar una u otra Universidad-IES/Dependencia en alguna de esas 4 categorías sea su total realidad en esta temática, ya que: «no siempre en los sitios web se publica-informa todo lo que se hace» o por el contrario «se publicainforma más de lo que realmente se hace»; por tanto, este tipo de análisis es una fotografía en un momento y desde un ángulo determinado que lleva a una aproximación y sistematización útil como visión general del desarrollo que se estaría dando en la formación en ALFIN.

\section{Análisis y resultados de la información sobre formación en ALFIN de los sitos web de las bibliotecas-CRAI universitarias de España}

Para la realización de este análisis se eligió como fuente inicial de identificación de los sitios Web de las bibliotecas universitarias españolas el sitio CRUEREBIUN 5 que reúne 72 universidades tanto públicas como privadas.

A su vez, con el fin de adicionar otras bibliotecas-CRAI, especialmente las de IES privadas con presencia importante en la Web, buscando así alcanzar un universo completo de las Universidades-IES españolas ${ }^{6}$, se recurrió a una fuente

\footnotetext{
5 Ver: http://www.rebiun.org/bibliotecas.html.

${ }^{6}$ Ver: http://www.oei.es/homologaciones/espana.pdf.
} 
internacional como el Ranking Web de Universidades del Mundo del CCHS/ CSIC $^{7}$ y el Portal estudiantil: El Atillo. $\mathbf{c o m}^{8}$, desde los cuales, tras su respectiva depuración y eliminación de duplicados, se añadieron otras (59 instituciones) que no estaban reportadas en CRUE-REBIUN. La recopilación y depuración de estas dos fuentes generó finalmente un listado de 131 Universidades-IES españolas: 50 públicas y 81 privadas (Anexo 1) 9

Para la realización del análisis se utilizó una ficha considerando postulados del análisis de contenidos (White y Marsh, 2006), en la cual se registraban los datos básicos de cada biblioteca, su url, la información sobre ALFIN teniendo en cuenta las 7 variables a valorar, especialmente la de Estructuración del programa, y, finalmente, considerando esa información, en cuál de los 4 niveles de incorporación de ALFIN se encontrarían.

Finalmente, tras el análisis de estas 131 bibliotecas-CRAI universitarias españolas según la información de sus sitios web, se obtuvieron estos resultados, que se sintetizan en las figuras 1 a 9 :

\section{FIGURA 1}

Bibliotecas universitarias españolas analizadas según comunidades

$$
(N=131)
$$

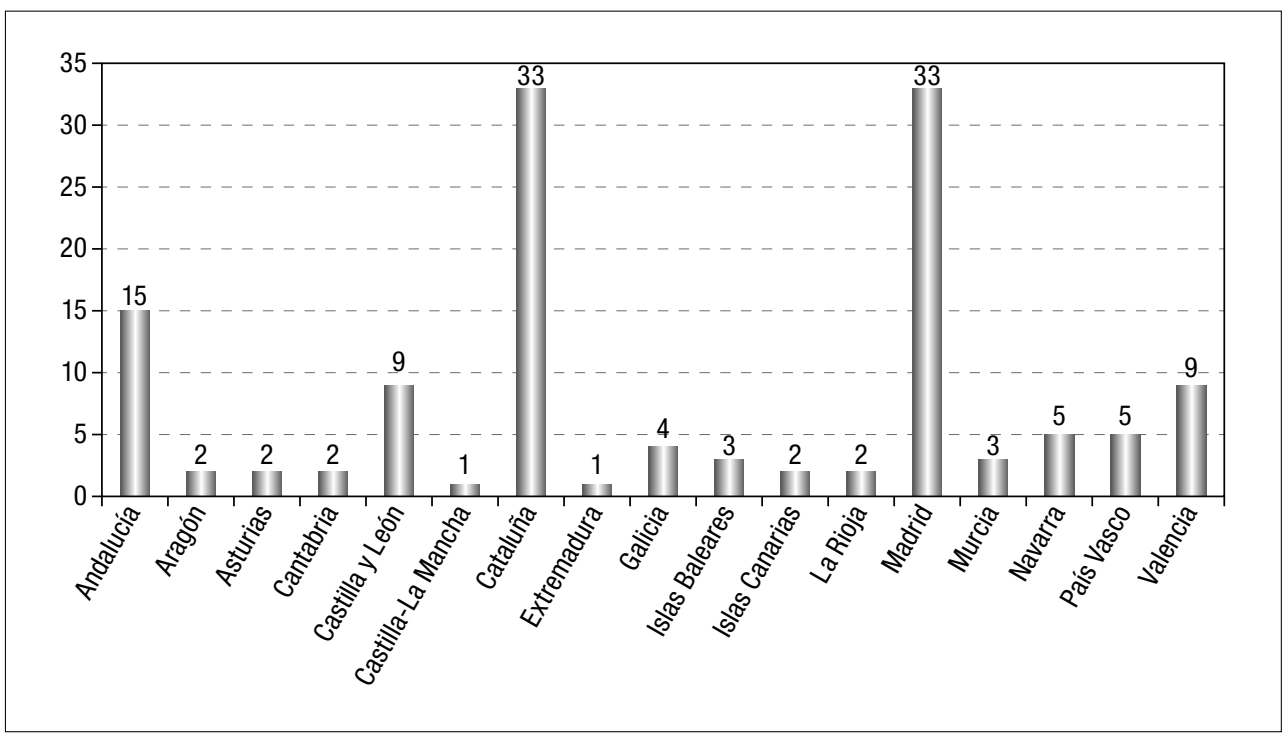

7 Ver: http://www.webometrics.info/rank_by_country_es.asp?country=es\&offset=0.

8 Ver: http://www.altillo.com/universidades/universidades_esp.asp.

9 El listado de las 131 IES españolas analizadas puede consultarse en línea en: http://bit.ly/zXp321. 


\section{FIGURA 2}

Bibliotecas universitarias que presentan información sobre algún nivel de formación $(N=131)$

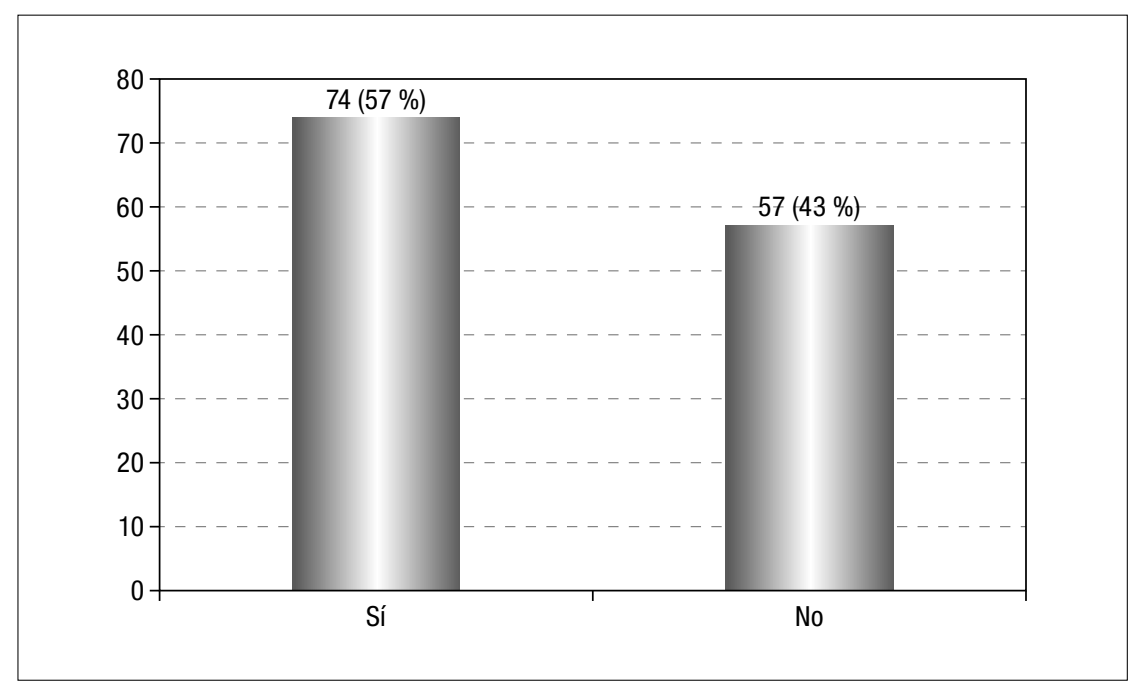

\section{FIGURA 3}

Niveles de incorporación de FU o ALFIN de las bibliotecas-CRAI universitarias españolas que presentan información en sus sitios web sobre actividades/cursos de formación $(N=74)$

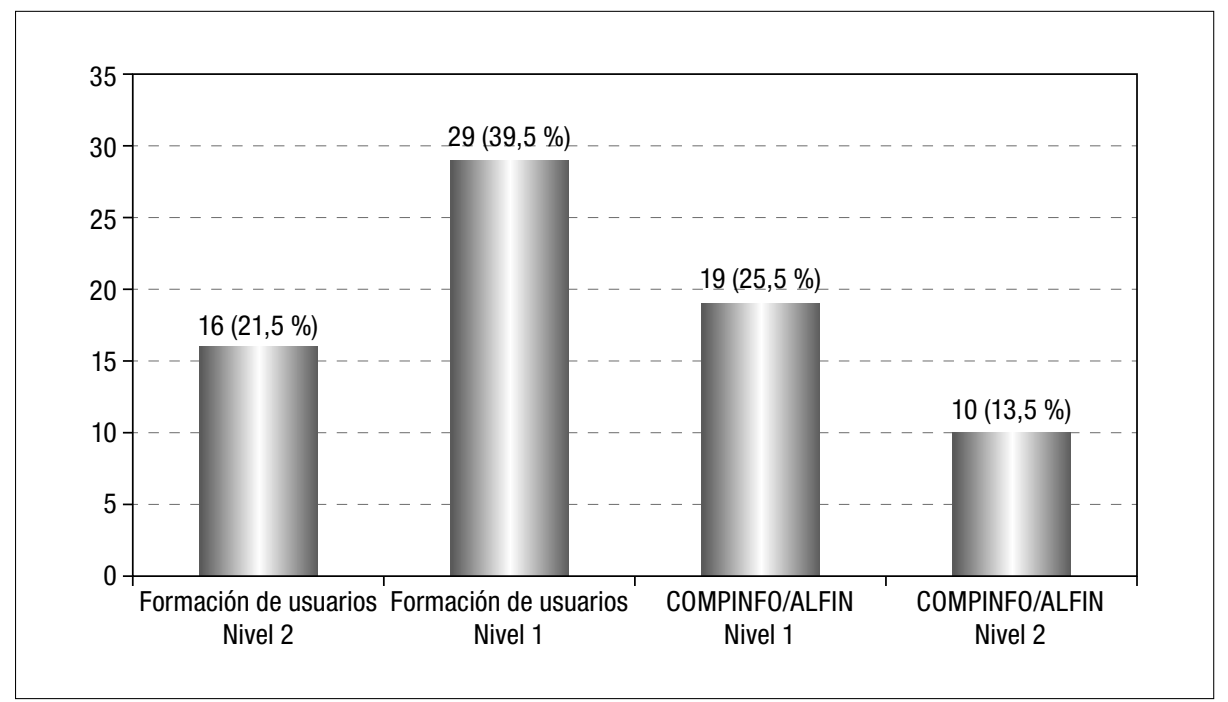




\section{FIGURA 4}

Niveles de incorporación de ALFIN 1 o 2 en universidades-IES españolas según tipología de universidad (pública o privada) $(N=29)$

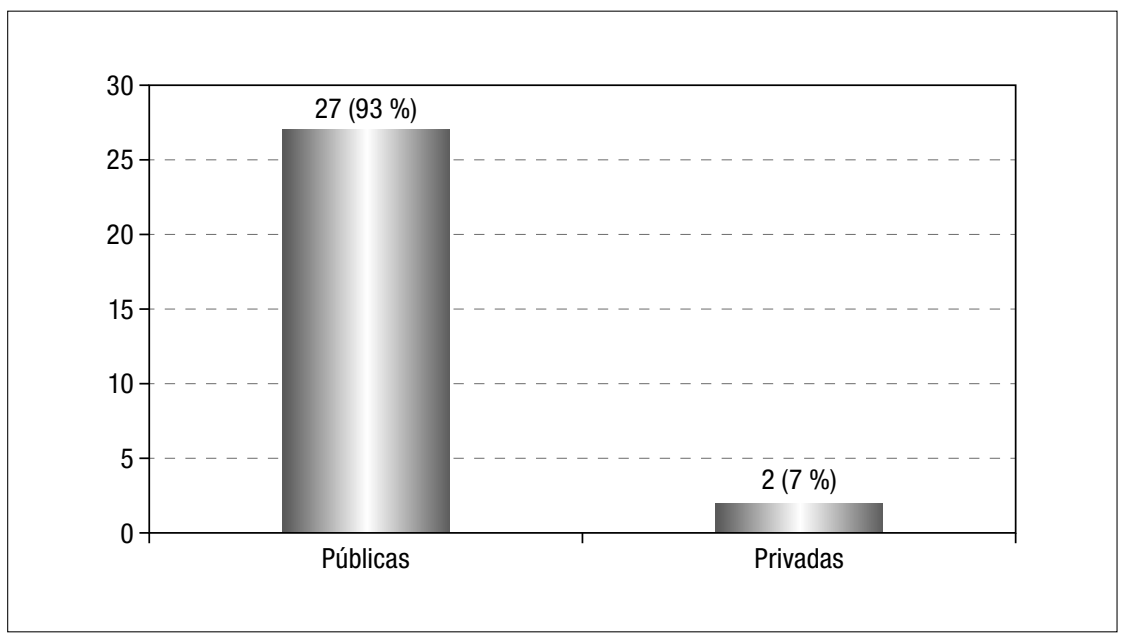

FIGURA 5

Porcentajes de incorporación en niveles 1 o 2 de ALFIN entre universidades públicas

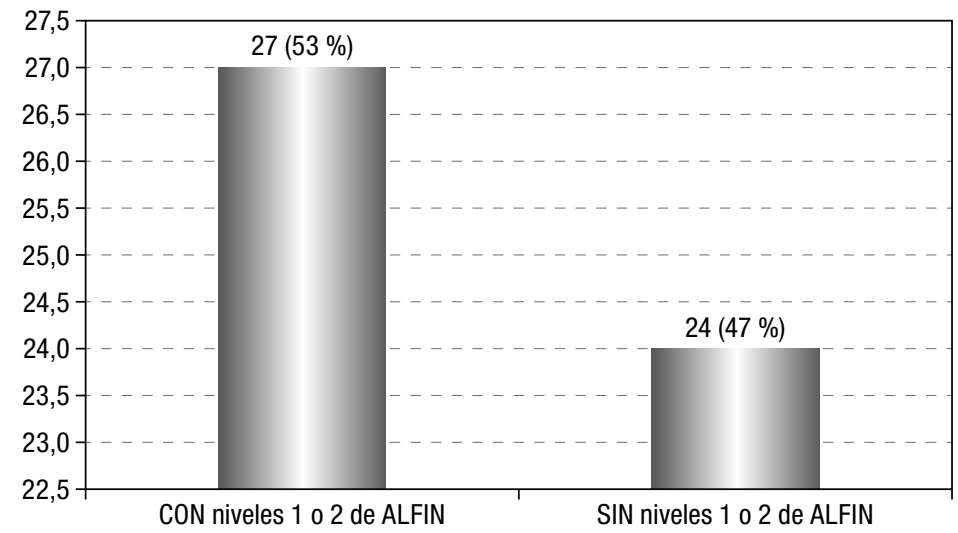




\section{FIGURA 6}

Porcentajes de incorporación en niveles 1 o 2 de ALFIN entre universidades privadas

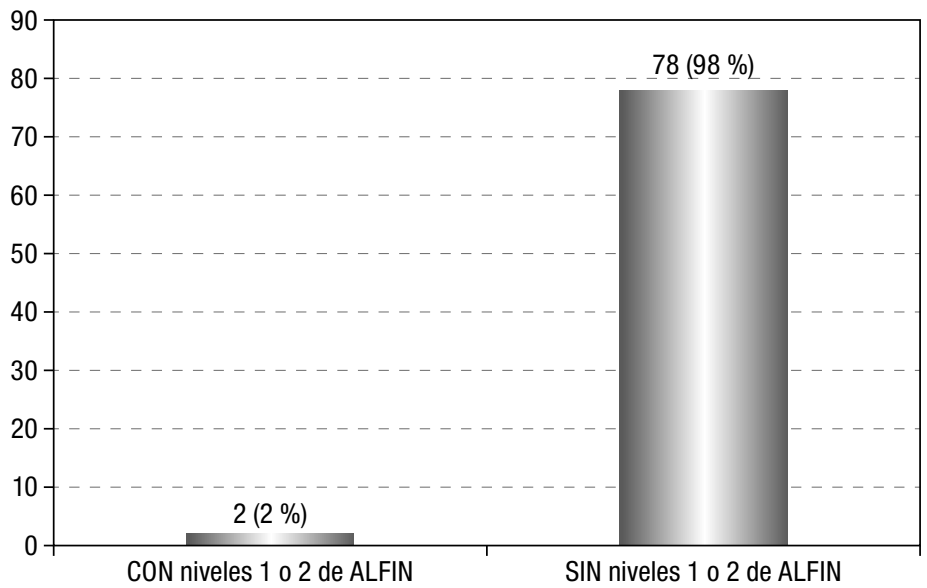

\section{FIGURA 7}

Procedencia según comunidades de las bibliotecas universitarias españolas que presentan información en sus sitios web de niveles de incorporación de ALFIN 1 o $2(N=29)$

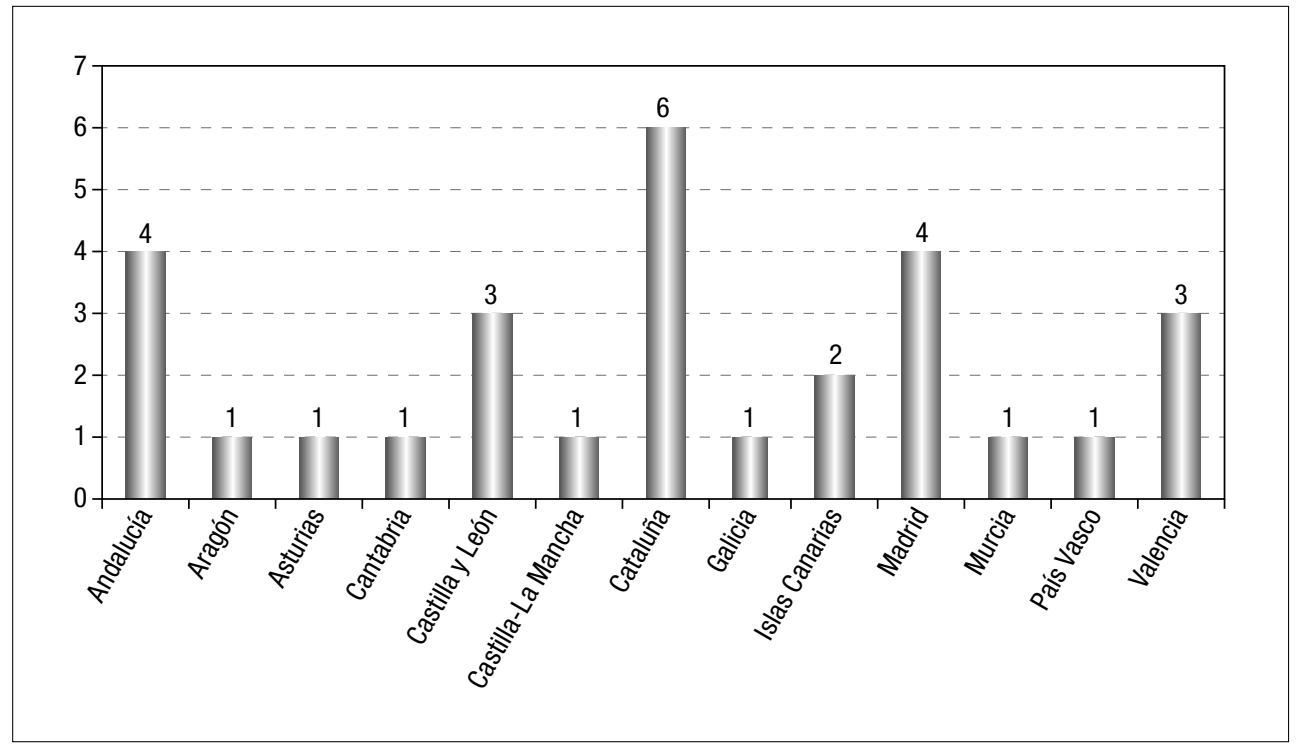




\section{FIGURA 8}

Porcentajes de presencia de Bibliotecas universitarias con niveles 1 o 2 de ALFIN según el total por comunidad

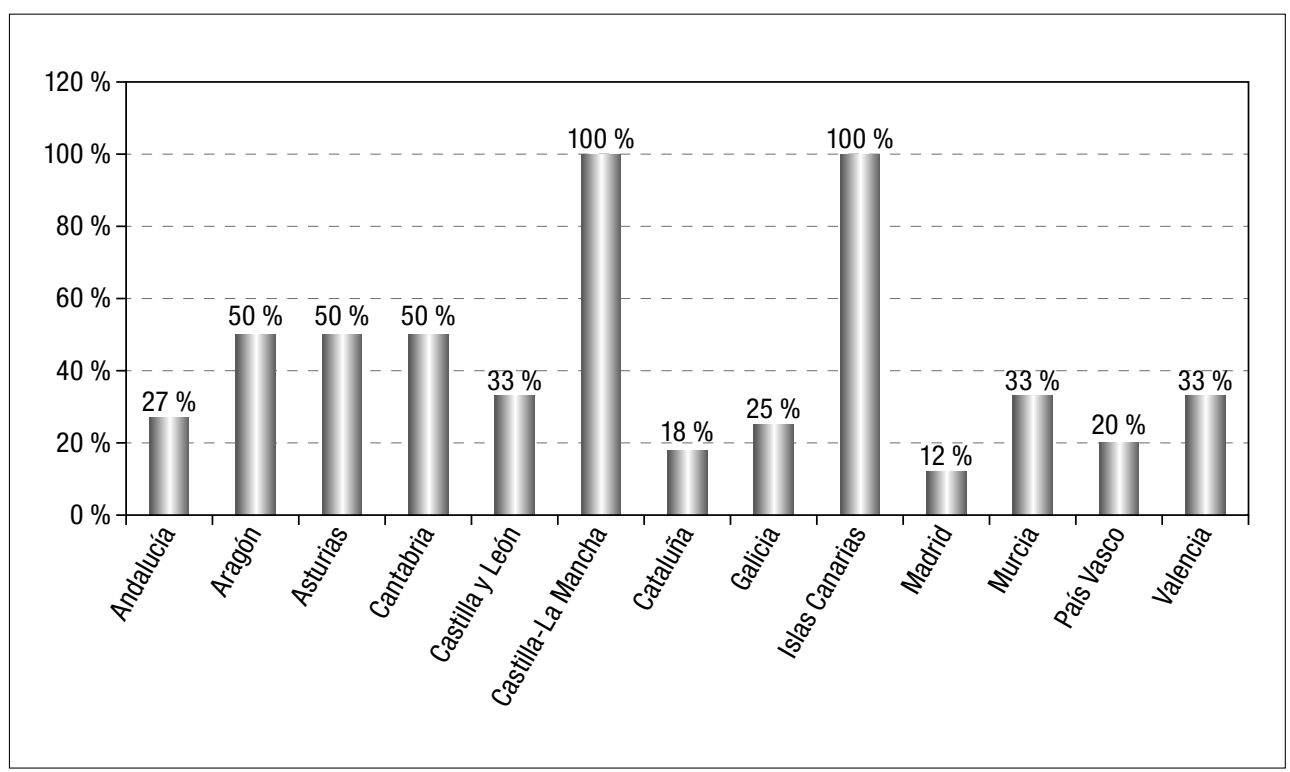

FIGURA 9

Relación entre universidades con niveles 1 o 2 de ALFIN y presencia de formación en biblioteconomía-documentación en esa universidad

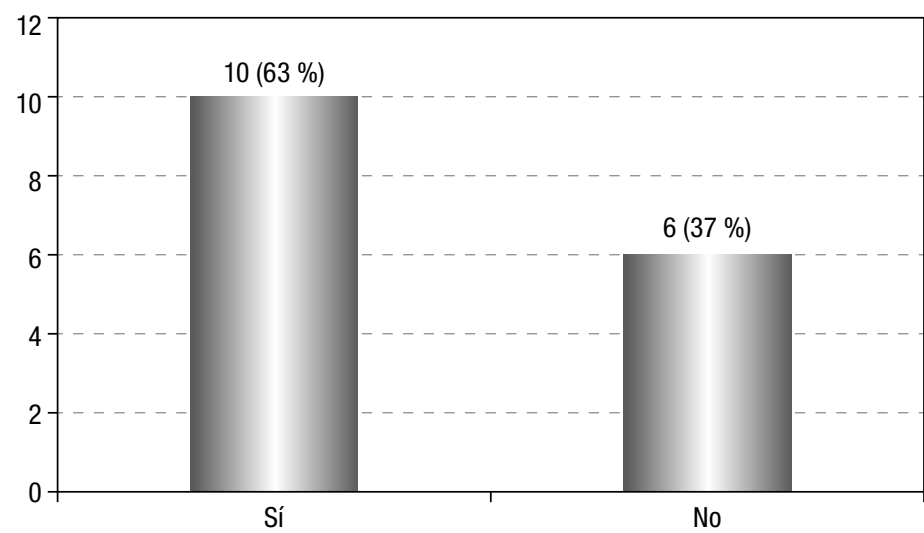




\section{Discusión y conclusiones}

Si partimos de dos premisas referentes a que:

- La Alfabetización Informacional, la formación en competencias informacionales, es una función constitutiva del papel que deben cumplir las bibliotecas-CRAI universitarias frente a los retos de la Educación Superior de hoy, considerando los requerimientos de la Sociedad de la Información; y

- La información que se publica en la Web, ante la nueva generación de usuarios (.Net, Google, 2.0, según las distintas denominaciones que se les han dado los últimos años) es un aspecto fundamental para que todos los servicios de las bibliotecas-CRAI se conozcan y su posicionamiento aumente como mediadoras de la información con valores agregados. Por tanto, si este medio no se gestiona de forma continua, si no hay una divulgación permanente, se está perdiendo una gran posibilidad de servicio a los usuarios que son la razón de ser de toda biblioteca.

Este estudio evidencia (figura 2) con preocupación, que en el caso de las bibliotecas-CRAI universitarias españolas hay un número-porcentaje considerable de instituciones (57-43\%) en las que ambas premisas o una de ellas no se estaría cumpliendo, ante lo que dichas instituciones deberían orientar su atención, teniendo en cuenta los efectos en la calidad de la educación que tiene la ALFIN. Es decir, o estas instituciones desde sus bibliotecas-CRAI no están realizando ningún o casi ningún tipo de actividad de formación (sea en algún nivel de F. U. o ALFIN), o si lo están haciendo, no se entiende el por qué no se divulga en su sitio Web ya que hay siempre que considerar como dicen algunos eslogan publicitarios: "Si no se divulga, no se conoce. Si no se conoce, no se usa. Si no se usa, tiene el peligro de desaparecer por no considerarse útil».

No obstante, ese sería un lado "negativo» o a mejorar que indica este estudio, pero si nos centramos en las Universidades-IES que sí presentan información sobre algún nivel de Formación (F. U. - ALFIN), es de destacar (figura 3) que 29 instituciones (39\%) ya estén en niveles 1 o 2 de ALFIN según las categorías que son consideradas en esta investigación. Cuando se analizan específicamente las bibliotecas-CRAI universitarias que están en estos niveles, se hace evidente la labor que los últimos años se ha venido haciendo considerando los retos que ha implicado Bolonia y los beneficios del trabajo conjunto, teniendo en cuenta que todas hacen parte de REBIUN-CRUE y que han sido las bibliotecas-CRAI que han liderado y tenido una participación constante en las Jornadas de CRAI (CRUETIC) y ALFIN REBIUN, e incluso algunas, impulsando desarrollos conjuntos con otras IES ${ }^{10}$.

Otro elemento a considerar, como reto, es que en las Universidades-IES privadas esta formación que alcance niveles 1 o 2 de ALFIN aún es un tema lejano,

${ }^{10}$ Ver: http://www.alfared.org/blog/bibliotecas-universitarias/1212. 
ya que solo 2 instituciones de esta tipología de universidad-IES muestran trabajos en esta línea en sus sitios web (figuras 4 y 6). Es un reto, ya que el mundo empresarial actual, al que se dirigen la mayoría de las veces estas instituciones, está siendo cada vez más exigente con sus nuevos profesionales esperando que los mismos cuando egresen, cuando sean nuevos empleados o emprendedores de negocios, tengan las competencias informacionales que les permitan interactuar adecuadamente en procesos de inteligencia competitiva, bechmarking, gestión del conocimiento, entre otros. Por tanto, la Universidad-IES que no evidencie estos logros formativos se irá poco a poco quedando relegada en el mercado laboral de sus egresados. La formación en ALFIN implica invertir en recursos documentales, de infraestructura, tecnológicos y de capital humano, pero a futuro tiene retribuciones tanto por el mejoramiento de la calidad de la educación como en competitividad e innovación, y esto es la razón de ser de cualquier universidad-IES, sea pública o privada.

Por otro lado, otro reto y oportunidad aparece también para las UniversidadesIES públicas españolas que aún están en procesos más de F. U. en nivel 1 o 2 (24 de 51 que hacen parte de esta tipología), ya que como se está viviendo ante los nuevos Grados, que incorporaron estas competencias informacionales en muchos casos de manera más formal (escrita) que aplicada, el hecho que otras 27 instituciones universitarias de su misma tipología estén ya en niveles de ALFIN (figuras 4 y 5) es una oportunidad para seguir creciendo y pasar a la práctica como colectivo completo de universidades públicas españolas: hacer bechmarking, establecer convenios de cooperación; realizar capacitaciones interinstitucionales; intercambiar experiencias de enseñanza-aprendizaje, compartir plataformas virtuales y objetos de aprendizaje, etc.

Reto y oportunidad que a su vez, se amplía a REBIUN y las 20 instituciones privadas asociadas que aún no estarían en esos niveles, para que prontamente los alcancen y así también impulsen a las otras IES privadas en relación con lo indicado en la conclusión anterior.

Finalmente, es importante resaltar la labor de un buen número de universidades que pertenecen a una misma Comunidad Autónoma (figura 7) con niveles 1 o 2 de ALFIN, ya que ello implicaría que su trabajo está haciendo y hará en el futuro que sus Comunidades se destaquen tanto en sus egresados como en el posicionamiento de sus bibliotecarios y bibliotecas-CRAI, especialmente en aquellas donde el número de universidades con estos niveles implica un porcentaje alto en relación con el total de universidades por Comunidad (figuras 1 y 8). Sin embargo, hay que considerar un mayor reto para determinadas Comunidades, ya que entre mayor número de universidades más arduo el trabajo colaborativo necesario para que todas las Universidades-IES de dicha comunidad se beneficien de los aportes de la formación en competencias informacionales.

A su vez, estos resultados para esas comunidades, tienen en muchos casos, relación directa con la presencia de determinadas Asociaciones Bibliotecarias Españolas y/o Escuelas-Facultades de Biblioteconomía-Documentación (figura 9) con profesionales y docentes-investigadores destacados en esta temáti- 
$\mathrm{ca}^{11}$, llevando a que desde las mismas haya un trabajo valioso tanto en la formación-actualización de los asociados como en docencia e investigación y sus respectivos aportes a los programas de ALFIN de sus bibliotecas-CRAI.

Para concluir este trabajo, es importante indicar que este estudio evidencia una realidad con el objetivo de aportar a los avances aplicados y teórico-conceptuales de esta temática, pero sobre todo, busca motivar el seguir trabajando colaborativamente por un mayor desarrollo de la alfabetización informacional en las universidades españolas, y desde el alcance macro de esta investigación, en las universidades de Iberoamérica, y por eso la importancia de identificarnos ${ }^{12}$, de reconocernos para trabajar en forma más mancomunada.

\section{Bibliografía}

Detlor, B.; Lewis, V. (2006). Academic Library Web Sites: Current Practice and Future Directions The Journal of Academic Librarianship, vol. 32 (3), 251-258.

EMPATIC (2009). Report on Current State and Best Practices in Information Literacy. European Commission: Lifelong Learning Programme.

http://empat-ic.eu/eng/Reports/D1.1-Report-on-Current-State-and-Best-Practices-in-Information-Literacy-Final [consultado: 11-12-2010].

Ferreira-Gomes, H.; Prudêncio, D. S.; da Conceição, A. V. (2010). A mediação da informação pelas Bibliotecas universitárias: um mapeamento sobre o uso dos dispositivos de comunicação na web. Informação \& Sociedade: Estudos, vol. 20 (3), 1-12.

http://www.ies.ufpb.br/ojs2/index.php/ies/article/view/9047/4812 [consultado: 9-01-2011].

Gardner, S. J; Juricek, J. E.; Xu, F. G. (2008). An Analysis of Academic Library Web Pages for Faculty. The Journal of Academic Librarianship, vol. 34 (1), 16-24.

Pinto-Molina, M.; Uribe-Tirado, A. (2011). Formación del bibliotecario como alfabetizador informacional. Anuario ThinkEPI.

http://www.thinkepi.net/anuario-thinkepi/anuario-thinkepi-2011 [consultado: 29-04-2011].

Sontang, G. (2011). Information literacy classification-Colombia. Facilitating Information Literacy Education.

http://www.linkedin.com/groupItem?view=\&gid=1850160\&type=member\&item=48506240 \&qid=b59f064e-713d-4286-b98d-fe572337839f\&trk=group_most_popular-0-bttl\&goback=\% 2Egmp_1850160 [consultado: 27-03-2011].

Uribe-Tirado, A. (2010a). Avances y perspectivas de ALFIN en Iberoamérica. Una mirada desde la publicación académico-científica y la web 1.0 y 2.0. Congreso INFO Cuba 2010 - Instituto de Información Científica y Tecnológica (IDICT), 1-30.

http://eprints.rclis.org/handle/10760/14638 [consultado: 21-02-2011].

11 Algunos de estos profesionales y profesores-investigadores se encuentran en: http://www. directorioexit.info/lista.php?directorio=exit\&relacion=materia\&campos[=directorios_exit_materia1. VA LOR::Educaci\%F3n\%2C+formaci\%F3n \%2C+alfin.

${ }^{12}$ Ver el blog ALFIN/Iberoamérica: http://alfiniberoamerica.blogspot.com/ y el Mapa de bibliotecas y proyectos ALFIN/Iberoamérica: http://bit.ly/9hu80u. 
Uribe-Tirado, A. (2010b). La Alfabetización Informacional en Iberoamérica. Una aproximación a su pasado, presente y futuro desde el análisis de la literatura publicada y los recursos web. IBERSID. Revista de sistemas de información y documentación. Universidad de Zaragoza. 2010, 165-176.

http://eprints.rclis.org/handle/10760/14638 [consultado: 21-02-2011].

Uribe-Tirado, A. (2010c). La Alfabetización Informacional en la Universidad. Descripción y Categorización según los Niveles de Integración de ALFIN. Caso Universidad de Antioquia. Revista Interamericana de Bibliotecología. vol. 33 (1), 10-45.

http://aprendeenlinea.udea.edu.co/revistas/index.php/RIB/article/view/6280/5801 [consultado: 21-02-2011].

Uribe-Tirado, A. (2011). Informe-Estado del Arte de la Alfabetización Informacional en Colombia. IFLA - Information Literacy Section. Marzo 2011. 26p.

http://www.ifla.org/en/publications/information-literacy-state-of-the-art-report-colombiaespa-ol [consultado: 21-02-2011].

Webber, S.; Johnston, B. (2006). Working towards the information literate university. En: Walton, G. and Pope, A. (Eds) Information literacy: recognising the need. Staffordshire University, Stoke-on-Trent. Oxford: Chandos. 47-58.

http://dis.shef.ac.uk/sheila/staffs-webber-johnston.pdf [consultado: 20-10-2009].

White, M. D.; Marsh, E. E (2006). Content Analysis: A Flexible Methodology. Library Trends, vol. (55) 1, 22-45. 


\section{Anexo 1. Listado de bibliotecas-CRAI de Universidades-IES españolas analizadas}

\begin{tabular}{|c|c|c|}
\hline Universidad-IES/Biblioteca-CRAI & $\begin{array}{l}\text { Pública/ } \\
\text { Privada }\end{array}$ & URL (Información sobre Formación) \\
\hline $\begin{array}{l}\text { 1. Barcelona Graduate School of Econo- } \\
\text { mics }\end{array}$ & Privada & $\begin{array}{l}\text { http://www.barcelonagse.eu/Library_Re- } \\
\text { sources.html }\end{array}$ \\
\hline 2. Casa de Velázquez & Privada & $\begin{array}{l}\text { http://www.casadevelazquez.org/es/bi- } \\
\text { blioteca/ }\end{array}$ \\
\hline 3. Centre Ernest Lluch UIMP & Privada & $\begin{array}{l}\text { http://www.cuimpb.cat/index. } \\
\text { php?option=com_remository\&Itemid= } \\
\text { 92\&lang=es }\end{array}$ \\
\hline 4. Centro de Estudios Financieros & Privada & http://www.cef.es/ \\
\hline $\begin{array}{l}\text { 5. Centro de Estudios Políticos y Consti- } \\
\text { tucionales }\end{array}$ & Privada & $\begin{array}{l}\text { http://www.cepc.es/ca/Recursos/Biblio- } \\
\text { teca.aspx }\end{array}$ \\
\hline $\begin{array}{l}\text { 6. Centro Internacional de Formación Fi- } \\
\text { nanciera }\end{array}$ & Privada & http://www.ciff.net/ \\
\hline 7. Centro Universitario Alberta Giménez & Privada & $\begin{array}{l}\text { http://www.cesag.org/es/servicios/bi- } \\
\text { blioteca.html }\end{array}$ \\
\hline 8. Centro Universitario Villanueva UCM & Privada & $\begin{array}{l}\text { http://www.villanueva.edu/biblioteca/ } \\
\text { servicios.html }\end{array}$ \\
\hline 9. CESINE Centro Universitario & Privada & $\begin{array}{l}\text { http://portal.cesine.com/conoce-cesine/ } \\
\text { bienvenido }\end{array}$ \\
\hline $\begin{array}{l}\text { 10. Colegio Universitario de Estudios Fi- } \\
\text { nancieros }\end{array}$ & Privada & $\begin{array}{l}\text { http://www.cunef.edu/departamentos/ } \\
\text { Departamentos/Biblioteca/Presentacion_ } \\
\text { c_229.html }\end{array}$ \\
\hline $\begin{array}{l}\text { 11. Conservatorio Superior de Música de } \\
\text { Salamanca }\end{array}$ & Privada & $\begin{array}{l}\text { http://www.consuperiorsal.com/index. } \\
\text { php? option=com_content \&task= } \\
\text { view\&id=159 }\end{array}$ \\
\hline $\begin{array}{l}\text { 12. Conservatorio Superior de Música Ma- } \\
\text { nuel Massotti Murcia }\end{array}$ & Privada & http://www.csmmurcia.com/ \\
\hline 13. CSIC & Pública & $\begin{array}{l}\text { http://bibliotecas.csic.es/formacion/ere- } \\
\text { cursos.html }\end{array}$ \\
\hline 14. Elisava Escola Superior de Disseny & Privada & $\begin{array}{l}\text { http://www.1961502011.net/ca/bibliote- } \\
\text { ca-enric-bricall }\end{array}$ \\
\hline 15. ESADE Business School Barcelona & Privada & $\begin{array}{l}\text { http://www.esade.edu/biblio/esp/servi- } \\
\text { cios/Formacioneng }\end{array}$ \\
\hline $\begin{array}{l}\text { 16. Escola d'Hoteleria de les Illes Balears } \\
\text { UIB }\end{array}$ & Privada & http://www.ehib.es/nova/ \\
\hline 17. Escola Superior de Disseny & Privada & $\begin{array}{l}\text { http://www.esdi.es/continguts/serveis. } \\
\text { php }\end{array}$ \\
\hline 18. Escola Superior de Música de Catalunya & Privada & $\begin{array}{l}\text { http://www.esmuc.cat/Biblioteca/Ser- } \\
\text { veis/Formacio-d-usuaris }\end{array}$ \\
\hline 19. Escola Sup. de Comerç Internacional & Privada & http://www.esci.es \\
\hline
\end{tabular}




\begin{tabular}{|c|c|c|}
\hline Universidad-IES/Biblioteca-CRAI & $\begin{array}{l}\text { Pública/ } \\
\text { Privada }\end{array}$ & URL (Información sobre Formación) \\
\hline $\begin{array}{l}\text { 20. Escola Universitària d'Hoteleria i Tu- } \\
\text { risme }\end{array}$ & Privada & $\begin{array}{l}\text { http://www.cett.es/cenrec/html/cat/ } \\
\text { labibl.html }\end{array}$ \\
\hline 21. Escola Universitària Salesiana de Sarria & Privada & $\begin{array}{l}\text { http://www.euss.es/web/portal/es/ } \\
\text { inicio.htm }\end{array}$ \\
\hline $\begin{array}{l}\text { 22. Escuela de Administración de Empresas } \\
\text { Barcelona }\end{array}$ & Privada & http://www.eae.es/es/servicios.html \\
\hline $\begin{array}{l}\text { 23. Escuela de Alta Direccion y Adminis- } \\
\text { tración }\end{array}$ & Privada & http://www.eada.edu/ \\
\hline 24. Escuela de Organización Industrial & Privada & $\begin{array}{l}\text { http://www.eoi.es/portal/guest/investiga- } \\
\text { cion/biblioteca }\end{array}$ \\
\hline 25. Escuela Europea de Negocios Madrid & Privada & http://www.een.edu/ \\
\hline 26. Escuela Internacional de Gerencia & Privada & $\begin{array}{l}\text { http://www.esgerencia.com/pag/16/club- } \\
\text { antiguos-alumnos }\end{array}$ \\
\hline 27. Escuela Técnica Lea-Artibai & Privada & $\begin{array}{l}\text { http://www.le artik.com/index. } \\
\text { php?Hizkuntza=ES }\end{array}$ \\
\hline 28. Escuela Universitaria de Osuna & Privada & $\begin{array}{l}\text { http://www. eu osuna.org/index. } \\
\text { php?option=com_content\&view=article\&i } \\
\text { d=28\&Itemid=42 }\end{array}$ \\
\hline $\begin{array}{l}\text { 29. Escuela Universitaria Padre Enrique de } \\
\text { Ossó }\end{array}$ & Privada & http://www.eupo.es/ \\
\hline $\begin{array}{l}\text { 30. ESIC Escuela Superior de Gestión Co- } \\
\text { mercial y Marketing }\end{array}$ & Privada & $\begin{array}{l}\text { http://www.biblioteca.esic.es/ABSYS/ } \\
\text { abwebp.exe/X5103/ID11451/G0 }\end{array}$ \\
\hline $\begin{array}{l}\text { 31. ETEA Institución Universitaria de la } \\
\text { Compañía de Jesús }\end{array}$ & Privada & http://www.etea.com/web/etea/servicios \\
\hline 32. Florida Universitaria & Privada & $\begin{array}{l}\text { http://www.florida-uni.es/web_es/servi- } \\
\text { cios/biblioteca-biblioteca-universitaria- } \\
\text { formacion/-/3/21/162/ }\end{array}$ \\
\hline $\begin{array}{l}\text { 33. Foro Europeo Escuela de Negocios de } \\
\text { Navarra }\end{array}$ & Privada & http://www.foroeuropeo.com/ \\
\hline 34. Fundació Universitària del Bages & Privada & $\begin{array}{l}\text { http://www.fub.edu/index.php?option= } \\
\text { com_content\&view=article\&id=43\&Item } \\
\text { id=1118 }\end{array}$ \\
\hline 35. Fundación IQS & Privada & http://biblioteca.iqs.es/ \\
\hline 36. I.E University & Privada & $\begin{array}{l}\text { http://www.ie.edu/universidad/students. } \\
\text { php?seccion=biblioteca\&contenido= } \\
\text { recursos_bibliografia }\end{array}$ \\
\hline $\begin{array}{l}\text { 37. IESE Business School Universidad de } \\
\text { Navarra }\end{array}$ & Privada & $\begin{array}{l}\text { http://www.iese.edu/en/Research/Li- } \\
\text { brary/TrainingSupport/TrainingSessions/ } \\
\text { TrainingSessions.asp }\end{array}$ \\
\hline 38. Instituciones Académicas Sandámaso & Privada & $\begin{array}{l}\text { http://www.fsandamaso.es/biblioteca_in- } \\
\text { terna.php?id=371 }\end{array}$ \\
\hline
\end{tabular}




\begin{tabular}{|c|c|c|}
\hline Universidad-IES/Biblioteca-CRAI & $\begin{array}{l}\text { Pública/ } \\
\text { Privada }\end{array}$ & URL (Información sobre Formación) \\
\hline $\begin{array}{l}\text { 39. Institut Barcelona d'Estudis Internacio- } \\
\text { nals }\end{array}$ & Privada & http://www.ibei.org/ \\
\hline $\begin{array}{l}\text { 40. Institut Nacional d'Educació Física de } \\
\text { Catalunya }\end{array}$ & Privada & $\begin{array}{l}\text { http://www.inefc.cat/inefc/AppPHP/bi- } \\
\text { blioteca.php?id_pagina=118 }\end{array}$ \\
\hline 41. Institut Universitari d'Estudis Europeus & Privada & $\begin{array}{l}\text { http://www.iuee.eu/index.asp?parent= } \\
\text { 0\&ap=1 }\end{array}$ \\
\hline 42. Instituto de Empresa Business School & Privada & $\begin{array}{l}\text { http://library.ie.edu/newsite/en/howtoci- } \\
\text { te.html }\end{array}$ \\
\hline 43. Instituto Europeo Campus Stellae & Privada & $\begin{array}{l}\text { http://www.campus-stellae.com/index. } \\
\text { html }\end{array}$ \\
\hline 44. Instituto Superior de Arte & Privada & http://www.iart.es/iart/ \\
\hline 45. Instituto Universitario de Posgrado & Privada & http://www.iup.es/ \\
\hline $\begin{array}{l}\text { 46. Instituto Universitario General Gutié- } \\
\text { rez Mellado UNED }\end{array}$ & Privada & http://iugm.es/quienes-somos/biblioteca/ \\
\hline 47. Mediterrani Escola de Turisme & Privada & $\begin{array}{l}\text { http://www.mediterrani.com/esp-univer- } \\
\text { sidad-turismo/m3-serveis-estudis/biblio- } \\
\text { teca.asp }\end{array}$ \\
\hline $\begin{array}{l}\text { 48. Musikene Centro Superior de Musica } \\
\text { del Pais Vasco }\end{array}$ & Privada & $\begin{array}{l}\text { http://www.musikene.net/castellano/in- } \\
\text { dex.asp }\end{array}$ \\
\hline 49. Real Centro Universitario María Cristina & Pública & $\begin{array}{l}\text { http://www.rcumariacristina.com/esp/ge- } \\
\text { neral.php?idApa=34 }\end{array}$ \\
\hline 50. Tecnocampus Mataró-Maresme & Privada & $\begin{array}{l}\text { http://www.tecnocampus.cat/web/estu- } \\
\text { dis-universitaris/serveis1 }\end{array}$ \\
\hline 51. Tecnun Universidad de Navarra & Privada & $\begin{array}{l}\text { http://www.tecnun.es/servicios/bibliote- } \\
\mathrm{ca} / \text { otros-servicios.html }\end{array}$ \\
\hline 52. Univaersitat de Girona & Pública & $\begin{array}{l}\text { http://www.udg.edu/biblioteca/LaBiblio- } \\
\text { tecaforma/tabid/12421/language/es-ES/ } \\
\text { Default.aspx }\end{array}$ \\
\hline 53. Universidad a Distancia de Madrid & Privada & http://www.udima.es/ \\
\hline 54. Universidad Alfonso X El sabio & Privada & $\begin{array}{l}\text { http://www.uax.es/ci/biblioteca.php/bi- } \\
\text { blioteca\# }\end{array}$ \\
\hline 55. Universidad Antonio de Nebrija & Privada & $\begin{array}{l}\text { http://www.nebrija.es/areas/biblioteca/ } \\
\text { formaciondeusuarios/formaciondeusua- } \\
\text { rios.html }\end{array}$ \\
\hline 56. Universidad Autónoma de Madrid & Pública & http://biblioteca.uam.es/ \\
\hline 57. Universidad Camilo José Cela & Privada & $\begin{array}{l}\text { http://www.ucjc.edu/index.php?section= } \\
\text { servicios/biblioteca/rincon-investigador }\end{array}$ \\
\hline 58. Universidad Cardenal Herrera & Privada & $\begin{array}{l}\text { http://www.uch.ceu.es/principal/biblio- } \\
\text { teca/informacion_general.asp?menuizqui } \\
\text { erda=biblioteca\&op=biblioteca\&menusu } \\
\text { perior= }\end{array}$ \\
\hline
\end{tabular}




\begin{tabular}{|c|c|c|}
\hline Universidad-IES/Biblioteca-CRAI & $\begin{array}{l}\text { Pública/ } \\
\text { Privada }\end{array}$ & URL (Información sobre Formación) \\
\hline 59. Universidad Carlos III de Madrid & Pública & $\begin{array}{l}\text { http://www.uc3m.es/portal/page/portal/ } \\
\text { biblioteca/aprende_usar }\end{array}$ \\
\hline 60. Universidad Católica de Ávila & Privada & $\begin{array}{l}\text { https://www.ucavila.es/_biblioteca/index_ } \\
\text { archivos/Page2081.htm }\end{array}$ \\
\hline $\begin{array}{l}\text { 61. Universidad Católica de Valencia San } \\
\text { Vicente Mártir }\end{array}$ & Privada & $\begin{array}{l}\text { https://www.ucv.es/alexandria/introduc- } \\
\text { cion.aspx }\end{array}$ \\
\hline $\begin{array}{l}\text { 62. Universidad Católica San Antonio de } \\
\text { Murcia }\end{array}$ & Privada & $\begin{array}{l}\text { http://www.ucam.edu/biblioteca/servi- } \\
\text { cios-1/gabinete-de-formacion }\end{array}$ \\
\hline 63. Universidad CEU Cardenal Herrera & Privada & $\begin{array}{l}\text { http://www.uchceu.es/servicios/bibliote- } \\
\text { ca.aspx?op=servicios }\end{array}$ \\
\hline 64. Universidad CEU San Pablo & Privada & http://www.campusvirtualceu.com/ \\
\hline 65. Universidad Complutense de Madrid & Pública & $\begin{array}{l}\text { http://www.ucm.es/BUCM/alfin/index. } \\
\text { php }\end{array}$ \\
\hline 66. Universidad de Alcalá & Pública & $\begin{array}{l}\text { http://www.uah.es/biblioteca/ayuda_for- } \\
\text { macion/Propiedad.html }\end{array}$ \\
\hline 67. Universidad de Alicante & Pública & $\begin{array}{l}\text { http://biblioteca.ua.es/es/puntbiu/tuto- } \\
\text { riales/formacion-en-linea-guias-y-tutoria- } \\
\text { les.html }\end{array}$ \\
\hline 68. Universidad de Almería & Pública & $\begin{array}{l}\text { http://cms.ual.es/UAL/universidad/servi- } \\
\text { ciosgenerales/biblioteca/servicios/servi- } \\
\text { cio/SERVICIO21772 }\end{array}$ \\
\hline 69. Universidad de Burgos & Pública & $\begin{array}{l}\text { http://www.ubu.es/ubu/cm/bubu/tkCont } \\
\text { ent;jsessionid=c192a00730d5af11d41755e } \\
\text { c40fab528b61324d6e880.e3uQahyRbxuN } \\
\text { e3iQc3aTb3eTay1ynknvrkLOlQzNp65In0 } \\
\text { ? i d C o n t e n t = 34941 \& l o c a l e = e s_ } \\
\text { ES\&textOnly=false }\end{array}$ \\
\hline 70. Universidad de Cádiz & Pública & http://www.uca.es/area/biblioteca/ \\
\hline 71. Universidad de Cantabria & Pública & $\begin{array}{l}\text { http://www.buc.unican.es/Servicios/for- } \\
\text { macion/formacion.asp }\end{array}$ \\
\hline 72. Universidad de Castilla la Mancha & Pública & http://biblioteca.uclm.es/tutoriales.html \\
\hline 73. Universidad de Córdoba & Pública & $\begin{array}{l}\text { http://www.uco.es/servicios/biblioteca/ } \\
\text { formacion/ayestudio.html }\end{array}$ \\
\hline 74. Universidad de Deusto & Privada & $\begin{array}{l}\text { http://www.biblioteca.deusto.es/servlet/ } \\
\text { Satellite/Page/1248091347530/_cast/\%231 } \\
\text { 107426114492\%231247725187273\%23124 } \\
\text { 8091347530/UniversidadDeusto/Page/Pa- } \\
\text { ginaCollTemplate }\end{array}$ \\
\hline 75. Universidad de Extremadura & Pública & $\begin{array}{l}\text { http://biblioteca.unex.es/servicios/forma- } \\
\text { cionusuarios.html }\end{array}$ \\
\hline 76. Universidad de Granada & Pública & http://biblioteca.ugr.es/pages/index \\
\hline
\end{tabular}




\begin{tabular}{|c|c|c|}
\hline Universidad-IES/Biblioteca-CRAI & $\begin{array}{l}\text { Pública/ } \\
\text { Privada }\end{array}$ & URL (Información sobre Formación) \\
\hline 77. Universidad de Huelva & Pública & $\begin{array}{l}\text { http://www.uhu.es/biblioteca/AyudaIn- } \\
\text { vestigador/AyudaInvestigador.htm }\end{array}$ \\
\hline 78. Universidad de Jaén & Pública & $\begin{array}{l}\text { http://www.ujaen.es/serv/biblio/forma- } \\
\text { cion/index_plata.html }\end{array}$ \\
\hline 79. Universidad de la Rioja & Pública & $\begin{array}{l}\text { http://biblioteca.unirioja.es/autoforma- } \\
\text { cion/index.shtml }\end{array}$ \\
\hline 80. Universidad de las Islas Baleares & Pública & $\begin{array}{l}\text { http://www.uib.es/ca/infsobre/serveis/ } \\
\text { generals/biblioteca/serveis_oferts/forma- } \\
\text { cio_usuaris/index.html }\end{array}$ \\
\hline $\begin{array}{l}\text { 81. Universidad de Las Palmas de Gran } \\
\text { Canaria }\end{array}$ & Pública & $\begin{array}{l}\text { http://biblioteca.ulpgc.es/formacion_ } \\
\text { cursos }\end{array}$ \\
\hline 82. Universidad de León & Pública & $\begin{array}{l}\text { http://www5.unileon.es/bibportal/servi- } \\
\text { cios/form_usu }\end{array}$ \\
\hline 83. Universidad de Málaga & Pública & http://www.uma.es/ficha.php?id=74091 \\
\hline 84. Universidad de Mondragón & Privada & $\begin{array}{l}\text { http://www.mondragon.edu/es/bibliote- } \\
\mathrm{ka} / \text { servicios/formacion }\end{array}$ \\
\hline 85. Universidad de Navarra & Privada & http://www.unav.es/biblioteca/ \\
\hline 86. Universidad de Oviedo & Pública & $\begin{array}{l}\text { http://www.uniovi.net/Biblioteca/ibipi/ } \\
\text { cursos_formacion/ }\end{array}$ \\
\hline 87. Universidad de Salamanca & Pública & http://sabus.usal.es/servicios.htm \\
\hline 88. Universidad de Santiago de Compostela & Pública & $\begin{array}{l}\text { http://busc.usc.es/Servizos/formacion. } \\
\text { asp }\end{array}$ \\
\hline 89. Universidad de Sevilla & Pública & $\begin{array}{l}\text { http://bib.us.es/aprendizaje_investiga- } \\
\text { cion/formacion/autoformacion-ides-id- } \\
\text { web.html }\end{array}$ \\
\hline 90. Universidad de Valladolid & Pública & $\begin{array}{l}\text { http://blogs.dmaweb.info/buva18/?page_ } \\
\text { id=107 }\end{array}$ \\
\hline 91. Universidad de Vigo & Pública & $\begin{array}{l}\text { http://webs.uvigo.es/servicios/bibliote- } \\
\mathrm{ca} / \text { referencia/Axudas.htm }\end{array}$ \\
\hline 92. Universidad de Zaragoza & Pública & $\begin{array}{l}\text { http://biblioteca.unizar.es/cursosforma- } \\
\text { cion.php }\end{array}$ \\
\hline 93. Universidad del País Vasco & Pública & $\begin{array}{l}\text { http://www.biblioteka.ehu.es/p207-con- } \\
\text { tent/es/contenidos/enlace/biblioteca_ } \\
\text { guias/es_guias/biblioteca_guias.html }\end{array}$ \\
\hline 94. Universidad Don Bosco & Privada & $\begin{array}{l}\text { http://www.cesdonbosco.com/profes/bi- } \\
\text { blioteca/default.html }\end{array}$ \\
\hline 95. Universidad Europea de Madrid & Privada & $\begin{array}{l}\text { http://biblioteca.uem.es/es/aprendizaje- } \\
\text { y-formacion }\end{array}$ \\
\hline $\begin{array}{l}\text { 96. Universidad Europea Miguel de Cer- } \\
\text { vantes }\end{array}$ & Privada & $\begin{array}{l}\text { http://www.uemc.es/es/Biblioteca/Inves- } \\
\text { tigacion/Paginas/Formacion.aspx }\end{array}$ \\
\hline
\end{tabular}




\begin{tabular}{|c|c|c|}
\hline Universidad-IES/Biblioteca-CRAI & $\begin{array}{l}\text { Pública/ } \\
\text { Privada }\end{array}$ & URL (Información sobre Formación) \\
\hline 97. Universidad Francisco de Vitoria & Privada & $\begin{array}{l}\text { http://www.ufv.es/vuniversitaria } \\
\text { aspx?sec=2441 }\end{array}$ \\
\hline $\begin{array}{l}\text { 98. Universidad Fundación San Pablo CEU } \\
\text { Andalucía }\end{array}$ & Privada & $\begin{array}{l}\text { http://www.ceuandalucia.com/biblioteca. } \\
\text { htm }\end{array}$ \\
\hline $\begin{array}{l}\text { 99. Universidad Internacional de Anda- } \\
\text { lucía }\end{array}$ & Pública & $\begin{array}{l}\text { http://www.unia.es/content/ } \\
\text { view/77/172/ }\end{array}$ \\
\hline 100. Universidad Internacional de la Rioja & Privada & $\begin{array}{l}\text { http://www.unir.net/universidad-online. } \\
\text { aspx }\end{array}$ \\
\hline 101. Universidad Internacional Valenciana & Privada & $\begin{array}{l}\text { http://www.viu.es/web/guest/universi- } \\
\text { dad/organización }\end{array}$ \\
\hline 102. Universidad La Laguna & Pública & $\begin{array}{l}\text { http://www.bbtk.ull.es/view/institucional/ } \\
\text { bbtk/Competencias_en_informacion/es }\end{array}$ \\
\hline $\begin{array}{l}\text { 103. Universidad Nacional de Educación a } \\
\text { Distancia U.N.E.D }\end{array}$ & Pública & $\begin{array}{l}\text { http://portal.uned.es/portal/page?_pa- } \\
\text { geid=93,1418355\&_dad=portal\&_} \\
\text { schema=PORTAL }\end{array}$ \\
\hline 104. Universidad Nebrija & Privada & $\begin{array}{l}\text { http://www.nebrija.com/servicios/biblio- } \\
\text { teca/index.htm }\end{array}$ \\
\hline 105. Universidad Oberta de Catalunya & Privada & http://biblioteca.uoc.edu/esp/index.html \\
\hline 106. Universidad Politécnica de Cartagena & Pública & $\begin{array}{l}\text { http://www.bib.upct.es/index.php/ } \\
\text { comp-inf }\end{array}$ \\
\hline 107. Universidad Politécnica de Madrid & Pública & $\begin{array}{l}\text { http://www.upm.es/institucional/UPM/ } \\
\text { Biblioteca/RecursosInformacion }\end{array}$ \\
\hline 108. Universidad Politécnica de Valencia & Pública & $\begin{array}{l}\text { http://www.upv.es/entidades/ABDC/in- } \\
\text { foweb/bg/info/712331normalc.html }\end{array}$ \\
\hline 109. Universidad Pontificia Comillas & Privada & $\begin{array}{l}\text { http://www.upcomillas.es/servicios/bi- } \\
\text { blioteca/serv_bibl_cont_tuto.aspx }\end{array}$ \\
\hline 110. Universidad Pontificia de Salamanca & Privada & $\begin{array}{l}\text { http://www.upsa.es/biblioteca/servicio/ } \\
\text { tutoriales/autoformacion.php }\end{array}$ \\
\hline 111. Universidad Privada Cardenal Cisneros & Privada & $\begin{array}{l}\text { http://www.cu-cisneros.es/alumnos. } \\
\text { php?sec=biblioteca\&sub=recursos }\end{array}$ \\
\hline 112. Universidad Pública de Navarra & Pública & http://www1.unavarra.es/biblioteca/ \\
\hline 113. Universidad Rey Juan Carlos & Pública & http://www.urjc.es/biblioteca/ \\
\hline 114. Universidad San Jorge & Privada & http://www.universidadsanjorge.net/ \\
\hline 115. Universidade da Coruña & Pública & $\begin{array}{l}\text { http://www.udc.es/biblioteca/galego/ } \\
\text { formacion.htm }\end{array}$ \\
\hline 116. Universitad de Lleida & Pública & $\begin{array}{l}\text { http://www.sbd.udl.cat/serveis/formacio. } \\
\text { html }\end{array}$ \\
\hline 117. Universitari de la Mediterrania & Privada & $\begin{array}{l}\text { http://www.campusmed.net/esp/quees. } \\
\text { php }\end{array}$ \\
\hline 118. Universitario EUSA & Privada & http://www.eusa.org.es/index.aspx \\
\hline
\end{tabular}




\begin{tabular}{|c|c|c|}
\hline Universidad-IES/Biblioteca-CRAI & $\begin{array}{l}\text { Pública/ } \\
\text { Privada }\end{array}$ & URL (Información sobre Formación) \\
\hline 119. Universitat Abat Oliba CEU & Privada & $\begin{array}{l}\text { http://www.uao.es/es/servicios/ } \\
\text { biblioteca?set_language=es }\end{array}$ \\
\hline 120. Universitat Autónoma de Barcelona & Pública & $\begin{array}{l}\text { http://www.uab.es/servlet/Satellite/bi- } \\
\text { bliotecas-1096479797005.html }\end{array}$ \\
\hline 121. Universitat de Barcelona & Pública & $\begin{array}{l}\text { http://www.ub.edu/web/ub/es/universi- } \\
\text { tat/serveis/biblioteca/biblioteca.html }\end{array}$ \\
\hline 122. Universitat de Valencia & Pública & $\begin{array}{l}\text { http://biblioteca.uv.es/castellano/servi- } \\
\text { cios/formacion_usuaris/ci2.php }\end{array}$ \\
\hline 123. Universitat de VIC & Privada & http://www.uvic.es/node/337 \\
\hline 124. Universitat Internacional de Catalunya & Privada & $\begin{array}{l}\text { http://www.uic.es/es/apoyo-investiga- } \\
\text { cion }\end{array}$ \\
\hline 125. Universitat Jaume I de Castellón & Pública & http://www.uji.es/CA/cd/cas/formacion/ \\
\hline 126. Universitat Politècnica de Catalunya & Pública & $\begin{array}{l}\text { http://bibliotecnica.upc.edu/content/ser- } \\
\text { vei-de-formaci\%C3\%B3-en-habilitats-in- } \\
\text { formacionals }\end{array}$ \\
\hline 127. Universitat Pompeu Fabra & Pública & $\begin{array}{l}\text { http://www.upf.edu/bibtic/es/serveis/ } \\
\text { sessions.html }\end{array}$ \\
\hline 128. Universitat Ramon Llull & Privada & $\begin{array}{l}\text { http://www.url.edu/es/cont/url/bibliote- } \\
\text { cas.php\#section1 }\end{array}$ \\
\hline 129. Universitat Rovira I Virgili & Pública & $\begin{array}{l}\text { http://www.urv.cat/biblioteca/serveis/ } \\
\text { formacio_usuaris.html }\end{array}$ \\
\hline 130. Univesidad Pablo de Olavide & Pública & $\begin{array}{l}\text { http://www.upo.es/biblioteca/bib_digi- } \\
\text { tal/index.jsp }\end{array}$ \\
\hline 131. Xarxa Vives d'Universitats & Privada & http://www.lluisvives.com/ \\
\hline
\end{tabular}

